\title{
Fabrication of isolated ferroelectric nanostructures
}

\author{
S. Bhattacharyya ${ }^{(a)}$, Soma Chattopadhyay ${ }^{(\bullet)}$ and M. Alexe \\ Max Planck Institute of Microstructure Physics \\ Weinberg 2, D-06120 Halle, Germany
}

\begin{abstract}
In this report, we explore the possibilities of the fabrication and characterization of ferroelectric nano-structures by various methods. Techniques include the self-assembly route and direct electron beam lithography. The chemical self-assembly route, which adopted the microemulsionmediated synthesis, appeared to be very encouraging for producing very small monodispersed nano particles and powders, with sizes of $10-60 \mathrm{~nm}$. The possibility to form a two dimensional arrangement of these micro droplets was explored with a suitable choice of the substrate material, and the method of coating. For the patterns prepared by the e-beam technique, a strong coupling between two closely spaced nano elements was observed, the spacing between them was found to be the key factor in tailoring their interaction, i.e., whether they would arrange themselves in rod-like pattern or a two dimensional array of isolated squares or circles.
\end{abstract}

\section{INTRODUCTION}

The quest for understanding nano particle processing methods is a natural consequence of the extensive activities carried out in the last decade that resulted in decreasing the size of electronic devices [1,2]. At present there are many ongoing research activities to explore the most rational and reproducible means of fabricating nano-patterns in a controlled manner [3,4]. Ferroelectrics are known to be a special class of functional materials that are promising for nano-scale applications such as in high density memory devices, miniaturized sensors and transducers $[5,6]$. It is therefore highly relevant to the scientific community to fabricate and study ferroelectric materials at the nanoscale level. In this paper, we are comparing the results of our attempts to fabricate ferroelectric nanostructures using both "top down" and "bottom up" approaches. The systems chosen were simple perovskites such as $\mathrm{BaTiO}_{3}$, and $\mathrm{Pb}\left(\mathrm{Zr}_{0.40}, \mathrm{Ti}_{0.60}\right) \mathrm{O}_{3}$, for their relative ease of fabrication. In the chemical "self assembly" route, we have employed the microemulsion mediated synthesis $[7,8]$ of monodispersed nano-particles (dispersed in a liquid medium). Although getting a regular arrangement of these particles on a solid surface is still in its infancy, it is possible to get isolated nano dots with reasonably uniform sizes. There was an agglomeration problem associated with the highly reactive fine particles which could be overcome by the method of peptization [9]. The "top down" method used was electron beam lithography, and the subsequent developing of appropriately prepared films. The substrate material, the electron beam intensity, the interval between the e-beam irradiated regions, and the choice of precursor were the deciding factors in the final size of the nano dots.

(a) Contact author: sudipta@mpi-halle.de

(•) CSRRI-IIT, MRCAT Sector 10, Advanced Photon Source, Building 433B, Argonne National Lab, Argonne, IL60439, USA. 


\section{EXPERIMENTAL.}

\section{Synthesis by the self assembly route:}

The microemulsion mediated synthesis involved the hydrolysis of the metallic alkoxides within nanosized water droplets in a cyclohexane/Brij 30 (Polyethylene glycol dodecyl ether) $/ \mathrm{H}_{2} \mathrm{O}$ water in oil medium, as suggested by Hempelmann and Herrig [8]. The microemulsion was first optimized for the maximum amount of water that could be accommodated in this system, and then, the microemulsion was slowly added to the appropriate amount of bimetallic precursor (Barium titanium ethylhexano-isopropoxide (Alfa Aeser), for $\mathrm{BaTiO} 3$ particles, for instance). The precursor reacted with the water droplets present in the microemulsion and formed the oxide particles. The reaction was confined within the water droplets. The mixture was then mechanically stirred for one hour until a slightly cloudy solution appeared. This solution was stable for a few weeks. The freshly prepared solution was heat dried to form a black residue which turned into a yellowish powder upon a further heat treatment $\left(450{ }^{\circ} \mathrm{C}\right.$ for 60 minutes). The as prepared powder was checked by SEM and XRD. To avoid agglomeration between the nano particles, we added tetrabutylammonium hydroxide as a peptizing agent.

\section{e-beam lithography:}

In the e-beam lithography process, a thin layer (approximately 70-100 nm thick after drying) of the precursor was deposited on a single crystalline strontium titanate (STO) substrate followed by a low temperature $\left(80^{\circ} \mathrm{C}\right)$ drying in order to evaporate the organic liquids. This sample was then exposed to a focussed electron beam programmed to hit the sample surface at regular spatial intervals. The interval was chosen to vary from $200 \mathrm{~nm}$ to $1 \mu \mathrm{m}$. The size of each exposed dot was also varied with the distance between them, as shown later. Two different solutions were chosen for the preparation of e-beam samples. The first one was a commercially available PZT precursor (from Chemat Corporation) and the other was a laboratory made solution, which was prepared by dissolving the individual metal alkoxides in a common solvent such as 2methoxyethanol and xylene.

\section{RESULTS AND DISCUSSION}

An SEM picture of the microemulsion-derived barium titanate powder is shown in figure 1. It could be seen that the as-dried powder was made of almost monodispersed particles with a size centered around $700 \mathrm{~nm}$. After ultrasonicating the specimens, the particles revealed themselves as well defined cubic grains with sizes of about $200-500 \mathrm{~nm}$ (Fig. 2). This external morphology of the grains confirmed the presence of perovskite $\mathrm{BaTiO}_{3}$. The XRD of the as prepared nano powder is shown in figure 3. It was evident from the result presented in Fig. 3 that the powder was phase pure $\mathrm{BaTiO}_{3}$. The formation of $\mathrm{BaTiO}_{3}$ phase at such low temperature $\left(\sim 450{ }^{\circ} \mathrm{C}\right)$ had been possible due of the enhanced reactivity of the nano-particles. However, there were a few grains that showed a fine structure, i.e. they looked like a cluster of much finer grains (Fig. 4). Each cluster was approximately $800 \mathrm{~nm}$ in size, which was close to the particle size in the as prepared powder (Fig. 1). The individual particles forming the agglomerates were of size less than $50 \mathrm{~nm}$. This was also a consequence of the ultra-fine grain structure of the as prepared particles which caused a significant increase in the number of unsaturated bonds terminating on their surfaces. It was therefore much more favorable for them to form larger agglomerates. These 


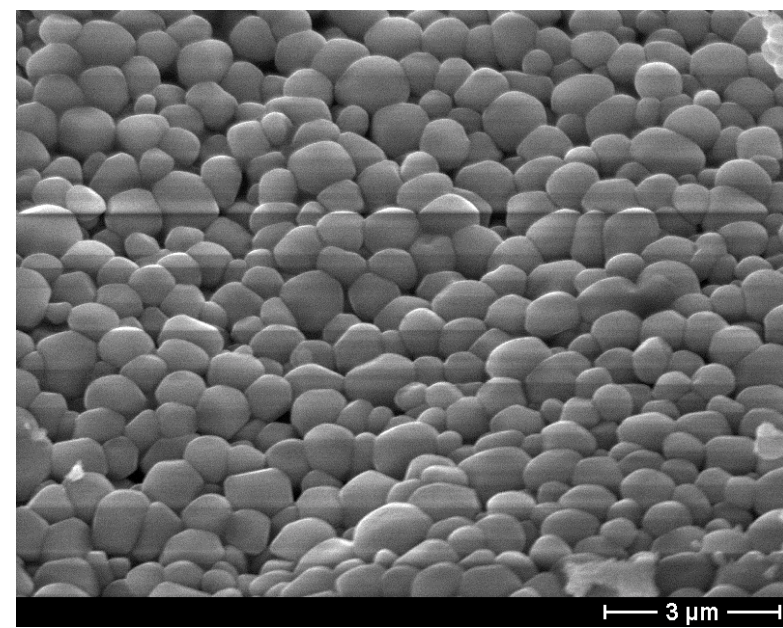

Figure 1. Microemulsion derived $\mathrm{BaTiO}_{3}$ nano powder (dried at $450^{\circ} \mathrm{C}$ )

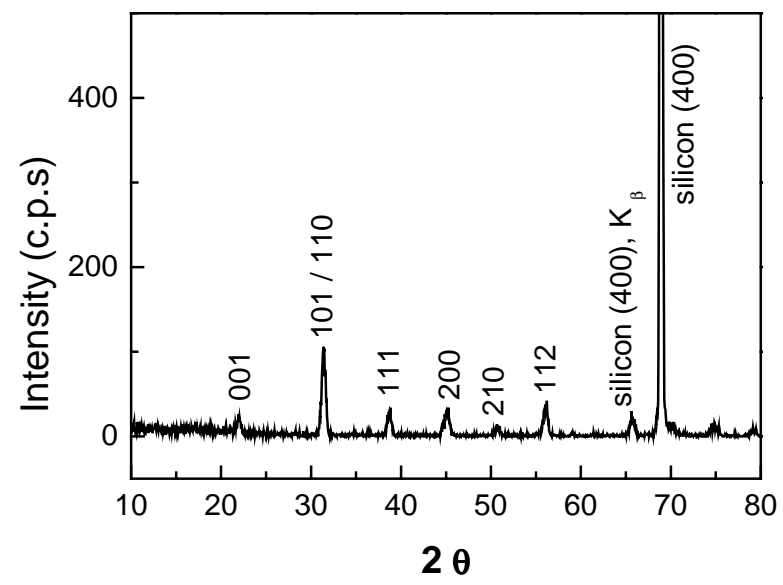

Figure 3. XRD of the $\mathrm{BaTiO}_{3}$ nanopowder. The strong peak at $69^{\circ}$ was from the silicon wafer on which the powder was glued.

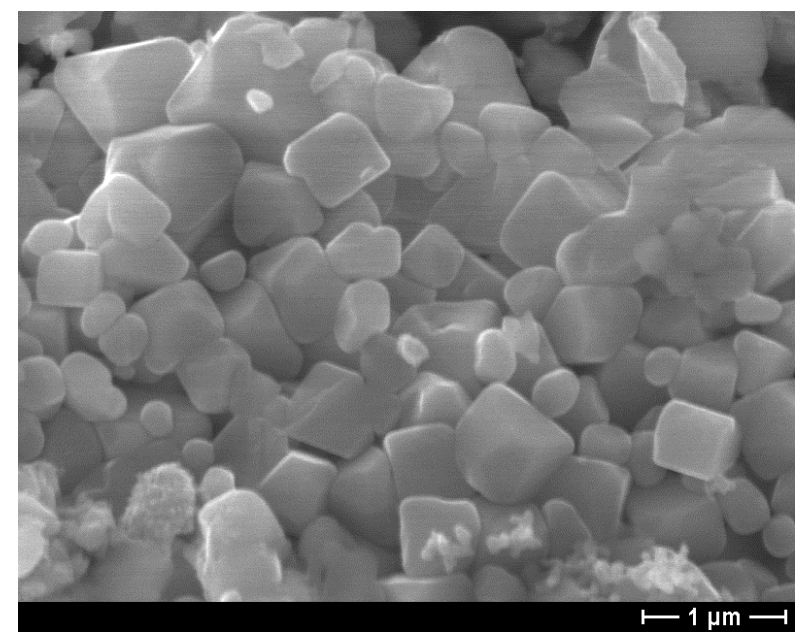

Figure 2. Dried $\mathrm{BaTiO}_{3}$ nano powder, after ultrasonic dispersion.

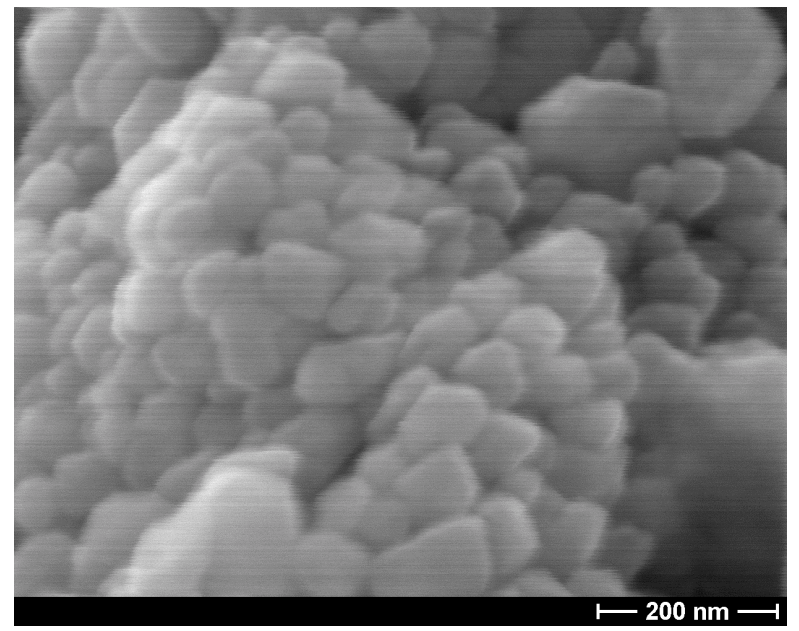

Figure 4. Agglomerated $\mathrm{BaTiO}_{3}$ nanoparticles.

finer particles were believed to be the product of the microemulsion mediated synthesis. However, it was seen that the precursor itself was also soluble in cyclohexane (without any surfactant). Therefore, it was thought that there could still have remained some amount of precursor dissolved in the liquid phase that were not intercepted by any water droplet. The bigger particles had most probably originated from the remaining (dissolved) precursor which could not be accommodated by the water droplets of the microemulsion. The as prepared microemulsion (before burning out the oil and surfactant) was coated on a bare silicon substrate followed by a low temperature annealing at $450{ }^{\circ} \mathrm{C}$ for 60 minutes. It was observed that the microemulsion formed a layer of interconnected particles on the silicon surface (as shown in Fig. 5). In the liquid phase, the equilibrium was maintained by the surface tensions of the oil and the surfactant. While drying, the oil phase evaporated first due to its lower boiling point, and the surfactant then drew the particles closer and formed the agglomerates. In order to remove the surfactants, the 


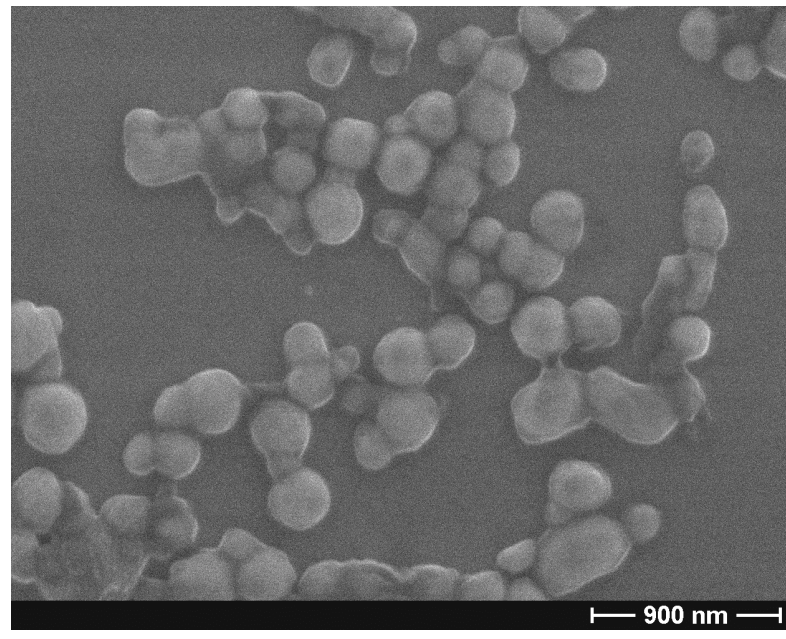

Figure 5. As prepared microemulsion sample coated on silicon and dried at $400^{\circ} \mathrm{C}$.

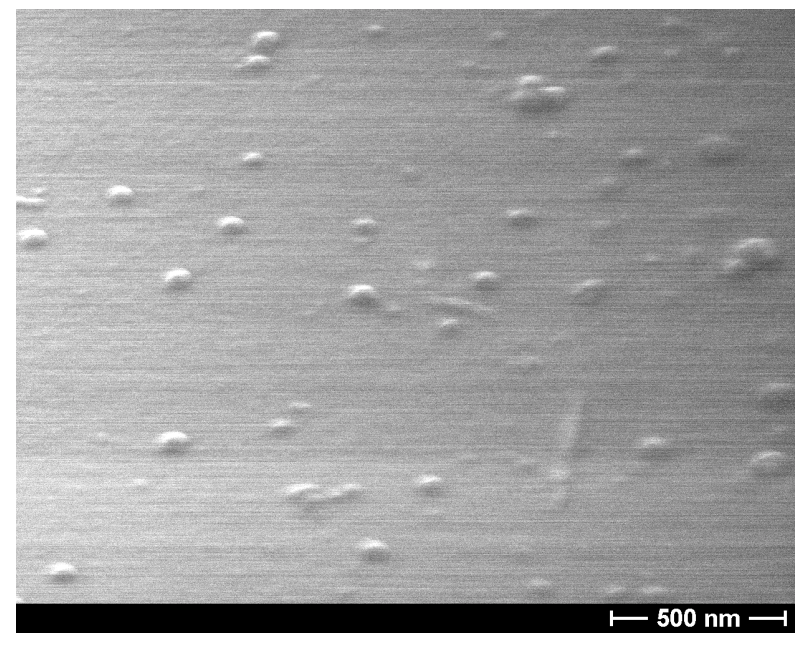

Figure 6. SEM image of the ultrasonicated microemulsion after coating \& annealing.

solution was washed with acetone followed by ultrasonic dispersion. This was further filtered, and coated on a silicon substrate again. After drying, the continuous layer revealed itself as made of well separated isolated particles. The SEM image of the sample coated with the ultrasonicated solution is shown in Fig. 6. It was observed that there was a distribution in the size of these particles, the average size was in the range of $50-60 \mathrm{~nm}$. The same solution was also coated on $\mathrm{Nb}$ doped $\mathrm{SrTiO}_{3}$ substrates with (001) orientation, and both SEM and AFM images were consistent with the previous observations, demonstrating that these particles were truly a result of the microemulsion assisted synthesis and not due to any artifact associated with the oxidation of the substrate. The heights of the microemulsion derived particles were in the range of 20-30 nm. There was however, no regularity observed in the positions of the nano droplets on the solid surface. The size of the particles were still too big for self assembly. The crystal field needs a size below $10 \mathrm{~nm}$ to lock the nano-dots at some preferred sites. Studies are in progress to achieve much finer particles on heat treated substrates.

While the microemulsion route promised a very fine nano structure, the e-beam lithography technique was the alternative to achieve a well defined array of nanoparticles with controllable size and inter-particle distance. Figure 7 shows the e-beam patterned nano-dots with different

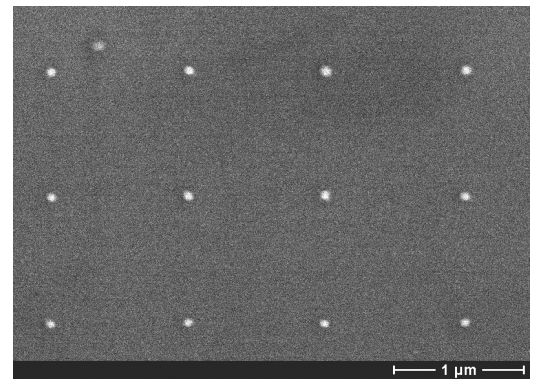

(a)

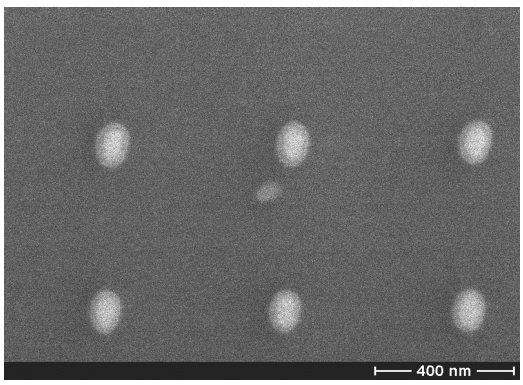

(b)

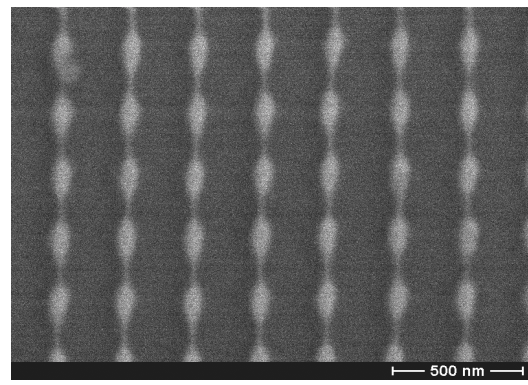

(c)

Figure 7. SEM images of the e-beam lithographic patterns as a function of the repetition distance (a) $1 \mu \mathrm{m}$, (b) $0.5 \mu \mathrm{m}$, and (c) $0.2 \mu \mathrm{m}$. 


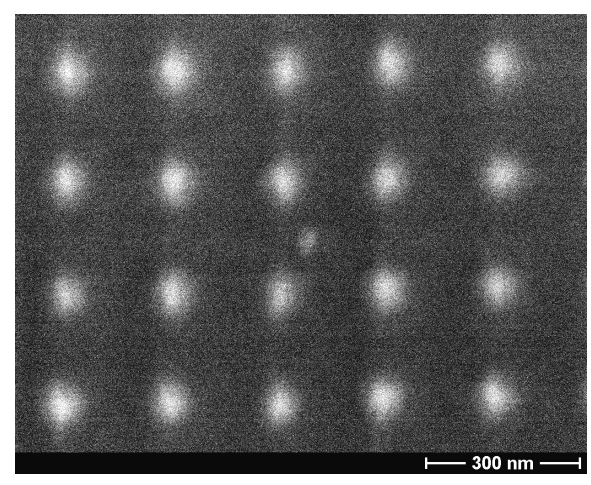

Figure 8. Patterns fabricated from the laboratory- prepared solution

intervals using a commercial solution. It can be seen from the micrographs that, for the case of the polymer precursor derived structure, there was a strong proximity effect between adjacent dots when they were placed at distances less than 0.5 micron. The dots did not remain as isolated dots, but joined together to form one dimensional arrays. The direction of the axis along which they joined was coincident with the e-beam scan direction. This was probably a result of the long-chain internal structure of the polymer solution. When such a solution was exposed to the ebeam, the exposed region would also affect the neighboring regions which were not exposed, but were lying on the same chain. This would limit the actual resolution of the e-beam lithography. On the other hand, figure 8 presents the image obtained from the e-beam pattern made from the prepared solution (containing individual precursors in the solvent matrix). It clearly demonstrated that one could get isolated nano-dots placed at regular intervals, even down to a size $\sim 150 \mathrm{~nm}$. It was demonstrated experimentally [10] for the case of PZT nano-dots that the as developed lithographic patterns change their shapes upon annealing at moderately high temperature $\left(\sim 650^{\circ} \mathrm{C}\right)$. It was thought to be a consequence of the enhanced surface energy contribution at smaller sizes. A similar observation was reported by Lange et al. [11] in which they showed that a continuous thin film goes through a shape-change upon annealing and breaks into smaller islands of pyramidal shape. Calculations based on purely thermodynamic considerations showed that, the system indeed lowered its energy while transforming to a pyramidal shape from an initially cubic shape [11]. A detailed calculation involving the growth kinetics of nano islands starting from an initially amorphous state is under way.

\section{CONCLUSIONS}

We have successfully synthesized using the microemulsion route phase-pure nano-powders as well as isolated nano-structures on solid substrates. The size was around $50 \mathrm{~nm}$. Studies are in progress in order to shrink the size further and to register these nanostructures in a regular fashion. On the other hand, the top down approach using e-beam lithography proved to be quite effective in fabricating regular arrays of ferroelectric nano-structures. The laboratory prepared solution was superior to the commercially available (polymer) solution, and could produce arrays of distinct nano-sized dots, the size being 60-70 $\mathrm{nm}$ which was due to the presence of shorter molecular chains present in the laboratory prepared solution. 


\section{ACKNOWLEDGEMENT}

Part of this work has been supported by the Volkswagen Stiffstung Project 'Nano-sized Ferroelectric Hybrids' under contract no. 5/77737.

\section{REFERENCES}

[1] Y. V. Nastaushev, T. Gavrilova, M. Kachanova, L. Nenasheva, V. Kolosanov, O. V. Naumova, V. P. Popov and A. L. Aseev, Materials Science \& Engineering C: Biomimetic Materials, Sensors \& Systems, C19, 189 (2002).

[2] J. Nishizawa, P. Plotka and T. Kurabayashi, IEEE Transactions on Electron Devices, 49, 1102 (2002).

[3] V. Millar, C. I. Pakes, A. Cimmino, D. Brett, D. N. Jamieson, S. D. Prawer, C. J. Yang, B. Rout, R. P. McKinnon, A. S. Dzurak and R. G. Clark, Proceedings of SPIE, 4590, 173 (2001).

[4] E. A. Guliants, C. Ji and W. A. Anderson, Journal of Electronic Materials, 31, 466 (2002).

[5] G. Braun, H. Hoenigschmid, T. Schlager, and W. Weber, J. Sol.-St. Circuits. 35, 691 (2000).

[6] V. Giurgiutiu, and Z. AN, Journal of Vibration \& Acoustics-Transactions of the ASME, 124, 116 (2002).

[7] M. Bonini, U. Bardi, D. Berti, C. Neto, P. Baglioni, J. Phys. Chem. B 106, 6178 (2002).

[8] H. Herrig and R. Hempelmann, Mat. Lett. 27, 287 (1996).

[9] J. Yang, S. Mei, J. M. F. Ferreira, Mat. Sci. and Engg. C 15, 183 (2001).

[10] M. Alexe, C. Harnagea, A. Vişnou, A. Pignolet, D. Hesse and U. Gösele, Scripta. Mater. 44, 1175 (2001).

[11] A. Seifert, A. Vojta, J. S. Speck, and F. F. Lange, J. Mat. Res., 11, 1470 (1996). 